Jorge E. Cortes and Hagop M. Kantarjian, The University of Texas MD Anderson Cancer Center, Houston, TX; Christine Powell, Pfizer, Cambridge, MA; Athena M. Countouriotis, Pfizer, La Jolla, CA; Dong-Wook Kim, Seoul St Mary's Hospital, Seoul, South Korea; Tim H. Brümmendorf, UniversitätsKlinikum Aachen, RheinischWestfaelische Technische Hochschule Aachen, Aachen; Tim H. Brümmendorf, Universitäts-Klinikum HamburgEppendorf, Hamburg, Germany; Irina Dyagil, Research Center for Radiation Medicine, Academy of Medical Sciences, Kiev, Ukraine; Laimonas Griskevicius, Vilnius University Hospital Santarškiu Klinikos, Vilnius University, Vilnius, Lithuania; Hemant Malhotra, Birla Cancer Centre, SMS Medical College, Jaipur, India; Karï Gogat, Pfizer Global Research and Development, Paris, France; and Carlo Gambacorti-Passerini, University Milano Bicocca, Monza, Italy.

Submitted August 9, 2011; accepted May 30, 2012; published online ahead of print at www.jco.org on September 4, 2012

Supported by Wyeth Research, which was acquired by Pfizer in October 2009

Clinical trial information: NCT00574873.

Presented in part at the 52nd Annua Meeting of the American Society of Hematology, December 4-7, 2010, Orlando, FL.

Authors' disclosures of potential conflicts of interest and author contributions are found at the end of this article.

Corresponding author: Jorge E. Cortes, $M D$, The University of Texas MD Anderson Cancer Center, 1515 Holcombe Blvd, Box 428, Houston, TX 77030; e-mail: jcortes@mdanderson .org

() 2012 by American Society of Clinical Oncology

0732-183X/12/3028-3486/\$20.00

DOI: $10.1200 / \mathrm{JCO} .2011 .38 .7522$

\title{
Bosutinib Versus Imatinib in Newly Diagnosed Chronic-Phase Chronic Myeloid Leukemia: Results From the BELA Trial
}

Jorge E. Cortes, Dong-Wook Kim, Hagop M. Kantarjian, Tim H. Brümmendorf, Irina Dyagil, Laimonas Griskevicius, Hemant Malhotra, Christine Powell, Karïn Gogat, Athena M. Countouriotis, and Carlo Gambacorti-Passerini

$$
\begin{array}{llllllll}
\text { A } & \text { B } & \text { S } & \text { T } & \text { R } & \text { A } & \text { C } & \text { T }
\end{array}
$$

\section{Purpose}

Bosutinib is an oral Src/Abl tyrosine kinase inhibitor. The phase III Bosutinib Efficacy and Safety in Newly Diagnosed Chronic Myeloid Leukemia (BELA) trial compared bosutinib with imatinib in newly diagnosed, chronic-phase chronic myeloid leukemia (CML).

\section{Patients and Methods}

A total of 502 patients were randomly assigned 1:1 to bosutinib 500 mg per day or imatinib 400 mg per day.

\section{Results}

The complete cytogenetic response (CCyR) rate at 12 months was not different for bosutinib $(70 \% ; 95 \% \mathrm{Cl}, 64 \%$ to $76 \%)$ versus imatinib $(68 \% ; 95 \% \mathrm{Cl}, 62 \%$ to $74 \%$; two-sided $P=.601)$; therefore, the study did not achieve its primary end point. The major molecular response (MMR) rate at 12 months was higher with bosutinib (41\%; 95\% Cl, 35\% to 47\%) compared with imatinib $(27 \% ; 95 \% \mathrm{Cl}, 22 \%$ to $33 \%$; two-sided $P<.001)$. Time to CCyR and MMR was faster with bosutinib compared with imatinib (two-sided $P<.001$ for both). On-treatment transformation to accelerated/blast phase occurred in four patients $(2 \%)$ on bosutinib compared with 10 patients $(4 \%)$ on imatinib. A total of three CML-related deaths occurred on the bosutinib arm compared with eight on the imatinib arm. The safety profiles of bosutinib and imatinib were distinct; $\mathrm{Gl}$ and liver-related events were more frequent with bosutinib, whereas neutropenia, musculoskeletal disorders, and edema were more frequent with imatinib.

\section{Conclusion}

This ongoing trial did not meet its primary end point of CCyR at 12 months, despite the observed higher MMR rate at 12 months, faster times to CCyR and MMR, fewer on-treatment transformations to accelerated/blast phase, and fewer CML-related deaths with bosutinib compared with imatinib. Each drug had a distinct safety profile.

\section{J Clin Oncol 30:3486-3492. ( 2012 by American Society of Clinical Oncology}

\section{INTRODUCTION}

Chronic myeloid leukemia (CML) is characterized by a constitutively active Bcr-Abl fusion protein, which is produced by a chromosomal translocation that forms the Philadelphia chromosome (Ph). ${ }^{1}$ Imatinib, a Bcr-Abl tyrosine kinase inhibitor (TKI), is currently indicated as first-line therapy of Ph-positive chronic-phase (CP) CML. ${ }^{2,3} \mathrm{~A}$ recent study of imatinib in newly diagnosed $\mathrm{CML}$ showed a correlation with major molecular response (MMR) by 12 months, including better progression-free survival (99\% $v 94 \%$ with no MMR; $P=.0023$ ) and improved overall survival (99\% v 93\% with no MMR; $P=.0011)$ at 3 years. ${ }^{4}$
In recent phase III studies, dasatinib and nilotinib have also demonstrated efficacy in newly diagnosed CP CML. ${ }^{5-9}$

Bosutinib (SKI-606) is an oral, dual Src/Abl TKI with more potent inhibitory activity against Bcr-Abl than imatinib in CML cell lines ${ }^{10,11}$ and minimal inhibitory activity against c-KIT or platelet-derived growth factor receptor, ${ }^{10,12} 2$ nonspecific targets potentially associated with toxicities reported for other second-generation TKIs. ${ }^{10,12-14}$ Bosutinib demonstrated clinical activity in patients with CP CML who had resistance or intolerance to prior TKI therapy. ${ }^{15,16}$ The current phase III trial (Bosutinib Efficacy and Safety in Newly Diagnosed Chronic Myeloid Leukemia [BELA]) compared the 
efficacy and safety of bosutinib with imatinib in newly diagnosed CP CML.

\section{PATIENTS AND METHODS}

\section{Patient Eligibility}

Eligible patients were adults with new ( $\leq 6$ months) diagnosis of Phpositive $\mathrm{CP} \mathrm{CML}^{17-19}$ who had received no prior antileukemia treatment (except $\leq 6$ months of anagrelide or hydroxyurea). Full eligibility criteria are listed in Appendix Table A1 (online only).

The study was conducted in accordance with the Declaration of Helsinki. All patients provided written informed consent, and the protocol was approved by institutional review boards at each site. The trial was registered at ClinicalTrials.gov (identifier: NCT00574873).

\section{Study Design and Treatment}

BELA is an open-label, randomized, multinational, phase III study. $\mathrm{Pa}-$ tients were randomly assigned 1:1 to bosutinib $500 \mathrm{mg}$ per day or imatinib 400 mg per day. Patients could continue study treatment until disease progression or early discontinuation. Patients were discontinued from treatment if they could not tolerate at least $300 \mathrm{mg}$ per day of bosutinib or imatinib. Dose escalation to $600 \mathrm{mg}$ per day of either bosutinib or imatinib was allowed for patients not achieving an optimal response per current European LeukemiaNet guidelines ${ }^{20}$ if no grade 3 or 4 or persistent grade 2 drug-related toxicity had been observed.

\section{Efficacy and Safety Assessments}

The primary efficacy end point was complete cytogenetic response (CCyR) at 12 months. Key secondary and exploratory efficacy end points included MMR, major cytogenetic response, and complete hematologic response (CHR) at 12 months; time to on-treatment transformation to accelerated phase (AP) or blast phase (BP) CML; time to first response; duration of response; response by Sokal risk group; on-treatment event-free survival (EFS); and overall survival (Appendix Table A2, online only).

Cytogenetic assessments were based on at least 20 metaphases or fluorescence in situ hybridization with at least 200 cells on peripheral blood or bone marrow (if cytogenetics were not available). Molecular assessments were based on quantitative reverse transcriptase polymerase chain reaction for Bcr-Abl in peripheral blood, all performed at one central laboratory (Molecular MD, Portland, OR). Molecular response was categorized as major (Bcr-Abl/Abl ratio of $\leq 0.1 \%$ on the International Scale) or complete (Bcr-Abl/Abl ratio of $\leq 0.01 \%$ on the International Scale with a sensitivity of $4.0 \operatorname{logs}$ ). The definition of CHR was standard ${ }^{15}$ and required confirmation after at least 4 weeks (Appendix Table A2). Assessments were performed every 3 months in the first year during study treatment.

Safety was assessed on an ongoing basis by physical examination (including vital signs), laboratory testing, and adverse event (AE) reporting. AEs were graded using the National Cancer Institute Common Terminology Criteria for Adverse Events, version 3.0.

\section{Statistical Analyses}

Response rates were calculated along with associated 95\% CIs for each treatment arm (Appendix Table A3, online only). These analyses were stratified by Sokal risk group (low, score $<0.8$; intermediate, score of 0.8 to 1.2; high, score $>1.2$ ) and geographic region based on a Cochran-MantelHaenszel test (two-sided $P$ value) unless an unstratified test based on the Fisher's exact test (two-sided $P$ value) was deemed more appropriate.

For key secondary and exploratory time-to-event end points, distributions and medians were estimated using the Kaplan-Meier method, and associated 95\% CIs for the medians were obtained using Brookmeyer-Crowley methodology. A Cox proportional hazards model stratified by Sokal risk group and geographic region was used to provide an estimate of the hazard ratio. All time-to-event end points are not yet mature, except time to response; duration of response is not reported, and no formal testing is available for other longterm end points at this time.
Efficacy analyses were performed on the intent-to-treat (ITT) population, which included all randomly assigned patients. Safety analyses were performed on the safety population, which included all patients who received at least one dose of study drug.

\section{RESULTS}

\section{Patients and Treatment}

A total of 502 patients with newly diagnosed CP CML were enrolled between February 2008 and July 2009, in 139 centers in 31 countries; 250 patients were randomly assigned to bosutinib, and 252 patients were randomly assigned to imatinib (Fig 1). Demographics and baseline disease characteristics are listed in Table 1. The current analysis was based on at least 12 months after the last patient's first visit. Median duration of treatment for both study arms was 13.8 months (bosutinib range, 0.03 to 30.3 months; imatinib range, 0.5 to 25.1 months). Median dose-intensities were $489 \mathrm{mg}$ per day for bosutinib (range, 115 to $575 \mathrm{mg}$ per day) and $400 \mathrm{mg}$ per day for imatinib (range, 201 to $542 \mathrm{mg}$ per day). During the study, nine patients (4\%) receiving bosutinib and 30 patients $(12 \%)$ receiving imatinib had their dose escalated to $600 \mathrm{mg}$ per day because of lack of efficacy. As of the data cutoff date, $71 \%$ of patients in the bosutinib arm and $80 \%$ in the imatinib arm were still receiving study treatment.

\section{Efficacy}

In the ITT population, the CCyR rate at 12 months was similar between patients receiving bosutinib (70\%; 95\% CI, 64\% to 76\%) and imatinib $(68 \% ; 95 \% \mathrm{CI}, 62 \%$ to $74 \% ; P=.601$; Fig $2 \mathrm{~A})$, with 21 patients ( $4 \%$; 10 patients on bosutinib; 11 on imatinib) included as having a CCyR at 12 months based on fluorescence in situ hybridization data only. Therefore, a superior CCyR rate at 12 months was not demonstrated for bosutinib versus imatinib, and the primary study end point was not met. However, median time to first CCyR was faster with bosutinib (12.9 weeks; 95\% CI, 12.6 to 13.4 weeks) compared with imatinib (24.6 weeks; 95\% CI, 24.3 to 25.6 weeks; $P<.001$ ), with higher rates of $\mathrm{CCyR}$ at months 3, 6, and 9 for bosutinib (Fig 2A). The cumulative rate of CCyR by 12 months was also similar between bosutinib and imatinib (79\%; 95\% CI, 74\% to $84 \%$ v 75\%; $95 \%$ CI, $69 \%$ to $80 \%$, respectively). The major cytogenetic response rate at 12 months was $73 \%$ for bosutinib and $78 \%$ for imatinib.

The MMR rate at 12 months was higher with bosutinib (41\%; $95 \%$ CI, $35 \%$ to $47 \%$ ) than imatinib (27\%; $95 \%$ CI, $22 \%$ to $33 \%$; $P<.001$; Fig $2 \mathrm{~B})$, with the complete molecular response rate at 12 months being $12 \%$ compared with $3 \%$, respectively $(P<.001)$. Median time to first MMR was faster with bosutinib $(37.1$ weeks; $95 \% \mathrm{CI}$, 36.1 to 48.6 weeks) compared with imatinib (72.3 weeks; 95\% CI, 61.1 weeks to not reached; $P<.001)$. The cumulative rate of MMR by 12 months was also higher with bosutinib (47\%; 95\% CI, 41\% to 53\%) versus imatinib (32\%; $95 \% \mathrm{CI}, 26 \%$ to $38 \%$; $P<.001)$.

The influence of Sokal risk group on treatment effect for CCyR and MMR at 12 months was further explored. The test for interaction showed that the association of either CCyR or MMR at 12 months with treatment did not demonstrate differences across Sokal risk groups $(P=.951$ and $P=.159$, respectively). CCyR rates at 12 months for bosutinib and imatinib were $78 \%$ and $75 \%$, respectively, in the low-risk group $(P=.623), 69 \%$ and $67 \%$, respectively, in the intermediate-risk group $(P=.708)$, and $56 \%$ for both treatment arms 


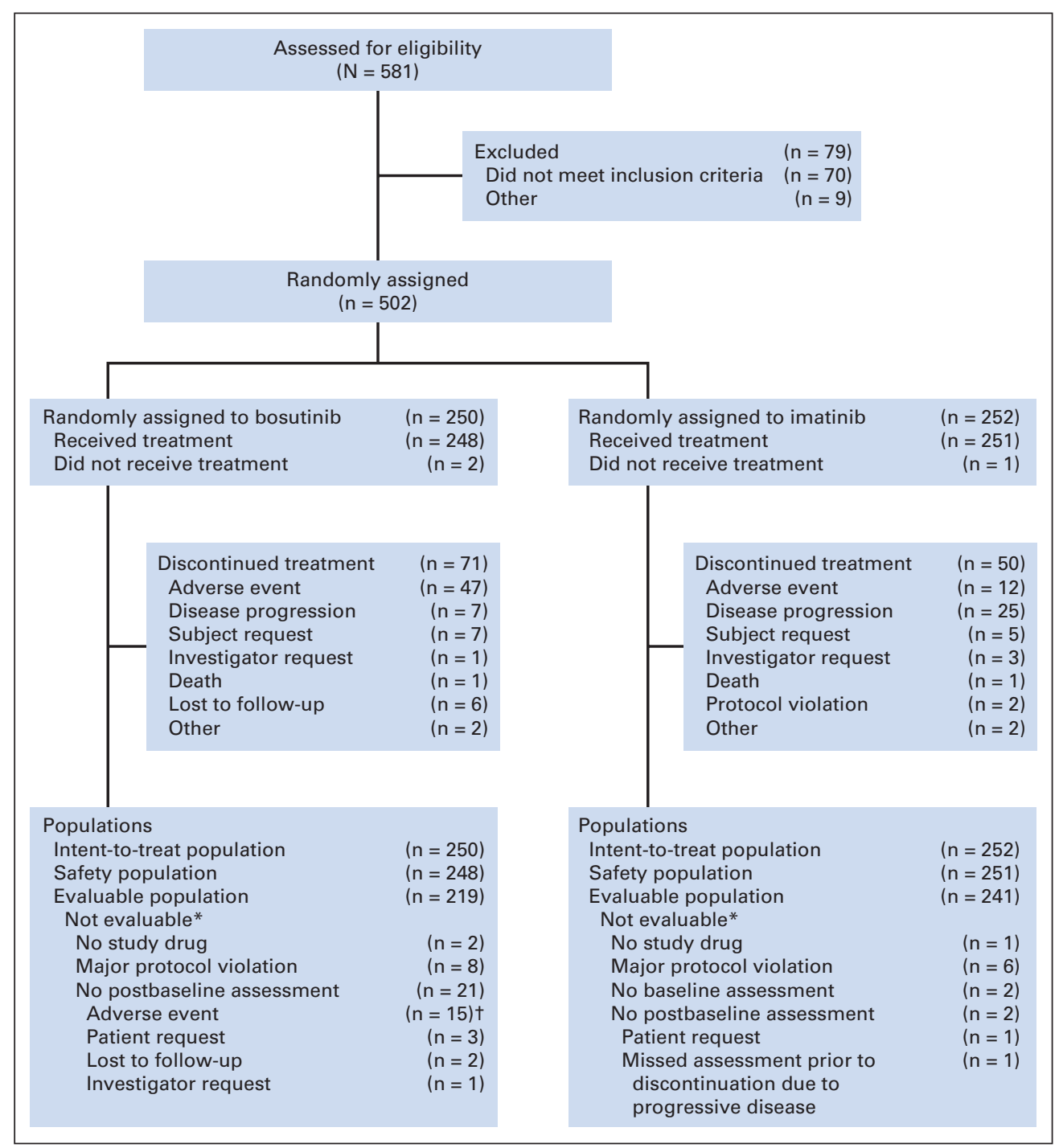

Fig 1. Disposition of patients. (*) Categories were calculated in sequential order to make the groups mutually exclusive. (†) Fifteen patients in the bosutinib arm had no postbaseline assessment because of adverse events, including elevated liver function tests $(n=5)$; vomiting ( $n=3)$; anaphylactic shock $(n=2)$; and dissociative disorder, dyspepsia, elevated lipase, decreased platelet count, and rash ( $n=1$ each)

in the high-risk group $(P>.999)$. MMR rates at 12 months were higher for bosutinib versus imatinib in the low-risk group (53\% v28\%, respectively; $P<.001$ ), intermediate-riskgroup (31\% $v 24 \%$, respectively; $P=.226)$, and high-risk group (33\% v28\%, respectively; $P=.651)$.

The rate of confirmed CHR at 12 months was $71 \%$ for bosutinib versus $85 \%$ for imatinib $(P>.999)$. Median times to first $\mathrm{CHR}$ were 4.4 weeks (95\% CI, 4.3 to 4.7 weeks) for bosutinib and 4.6 weeks (95\% CI, 4.4 to 5.0 weeks) for imatinib $(P=.5790)$.

Fewer patients had an on-treatment EFS event with bosutinib $(\mathrm{n}=11)$ than imatinib $(\mathrm{n}=18)$. Kaplan-Meier estimates of ontreatment EFS at 12 months were $94 \%$ for bosutinib compared with $93 \%$ for imatinib. Fewer patients in the bosutinib arm $(n=4,2 \%)$ experienced on-treatment transformation to AP/BP CML compared with the imatinib arm $(\mathrm{n}=10,4 \%)$. In an additional analysis of EFS that included death at any time or on-treatment transformation to AP/BP CML, six patients had an event in the bosutinib arm compared with 15 patients in the imatinib arm; these events consisted of seven patients reporting death (two patients $v$ five patients, respectively), seven patients reporting transformation to $\mathrm{AP} / \mathrm{BP}$ (two patients $v$ five patients, respectively), and seven patients reporting both events (two patients $v$ five patients, respectively). There were fewer deaths in the bosutinib arm ( $\mathrm{n}=4$; causes were CML related $[\mathrm{n}=3$ ] and mesenteric embolism/intestinal necrosis $[\mathrm{n}=1]$ ) compared with the imatinib arm $(n=10$; causes were CML related $[n=8]$, cardiovascular disease $[n=1]$, and lung embolism $[n=1]$; Fig 3). None of the deaths were considered related to study treatment. Kaplan-Meier estimates of overall survival at 12 months were greater than $99 \%$ in the bosutinib arm and $97 \%$ in the imatinib arm.

\section{Safety and Tolerability}

Treatment interruptions caused by AEs were required for 152 patients $(61 \%)$ receiving bosutinib and 105 patients (42\%) receiving imatinib; dose reductions as a result of AEs occurred for 97 patients (39\%) and 45 patients (18\%), respectively. Discontinuations as a result of AEs occurred in 48 patients (19\%) receiving bosutinib and 14 patients $(6 \%)$ receiving imatinib. Fifteen patients in the bosutinib arm discontinued treatment as a result of an $\mathrm{AE}$ (elevated aminotransferase $[\mathrm{n}=5]$, vomiting $[\mathrm{n}=3]$, anaphylactic shock $[\mathrm{n}=2]$, and dissociative disorder, dyspepsia, elevated lipase, decreased platelet count, and rash $[n=1$ each $]$ ) before their first postbaseline assessment at month 3 . 
Table 1. Patient Demographics and Baseline Disease Characteristics

\begin{tabular}{|c|c|c|c|c|}
\hline \multirow[b]{2}{*}{ Demographic or Characteristic } & \multicolumn{2}{|c|}{$\begin{array}{l}\text { Bosutinib } \\
(n=250)\end{array}$} & \multicolumn{2}{|c|}{$\begin{array}{l}\text { Imatinib } \\
(n=252)\end{array}$} \\
\hline & $\begin{array}{c}\text { No. of } \\
\text { Patients }\end{array}$ & $\%$ & $\begin{array}{c}\text { No. of } \\
\text { Patients }\end{array}$ & $\%$ \\
\hline \multicolumn{5}{|l|}{ Age, years } \\
\hline Median & \multicolumn{2}{|l|}{48} & \multicolumn{2}{|l|}{47} \\
\hline Range & \multicolumn{2}{|l|}{$19-91$} & \multicolumn{2}{|l|}{$18-89$} \\
\hline Sex, male & 149 & 60 & 135 & 54 \\
\hline \multicolumn{5}{|l|}{ Race } \\
\hline White & 160 & 64 & 164 & 65 \\
\hline Asian & 64 & 26 & 57 & 23 \\
\hline Other & 26 & 10 & 31 & 12 \\
\hline \multicolumn{5}{|l|}{ Hematologic analysis } \\
\hline \multicolumn{5}{|l|}{ WBC count, $10^{9} / \mathrm{L}$} \\
\hline Median & \multicolumn{2}{|l|}{21.7} & \multicolumn{2}{|l|}{23.5} \\
\hline 25th percentile & \multicolumn{2}{|l|}{10.6} & \multicolumn{2}{|l|}{11.3} \\
\hline 75th percentile & \multicolumn{2}{|l|}{56.3} & \multicolumn{2}{|l|}{58.0} \\
\hline \multicolumn{5}{|l|}{ Platelet count, $10^{9} / \mathrm{L}$} \\
\hline Median & \multicolumn{2}{|l|}{386} & \multicolumn{2}{|l|}{451} \\
\hline 25th percentile & \multicolumn{2}{|l|}{262} & \multicolumn{2}{|l|}{291} \\
\hline 75th percentile & \multicolumn{2}{|l|}{680} & \multicolumn{2}{|l|}{629} \\
\hline \multicolumn{5}{|l|}{ Time since diagnosis, days } \\
\hline Median & \multicolumn{2}{|l|}{23} & \multicolumn{2}{|l|}{22} \\
\hline Range* & \multicolumn{2}{|l|}{$0-183$} & \multicolumn{2}{|l|}{$0-241$} \\
\hline Interquartile range & \multicolumn{2}{|l|}{$9-45$} & $11-57$ & \\
\hline ECOG performance status & & & & \\
\hline 0 & 185 & 74 & 181 & 72 \\
\hline 1 & 65 & 26 & 71 & 28 \\
\hline Sokal riskt & & & & \\
\hline Low & 88 & 35 & 89 & 35 \\
\hline Intermediate & 117 & 47 & 118 & 47 \\
\hline High & 45 & 18 & 45 & 18 \\
\hline Geographic region & & & & \\
\hline $\begin{array}{l}\text { United States, Canada, western } \\
\text { EU, Australia }\end{array}$ & 65 & 26 & 66 & 26 \\
\hline $\begin{array}{l}\text { Eastern EU, Latin America, } \\
\text { South America }\end{array}$ & 77 & 31 & 79 & 31 \\
\hline $\begin{array}{l}\text { Other (eg, India, Japan, } \\
\text { Singapore) }\end{array}$ & 108 & 43 & 107 & 42 \\
\hline
\end{tabular}

Abbreviations: ECOG, Eastern Cooperative Oncology Group; EU, European Union. ${ }^{*}$ Range minimum is zero because of chronic myeloid leukemia diagnosis during the study screening period; range maximum is $>6$ months because of one patient who was considered a major protocol violator.

HLow corresponds to Sokal score of $<0.8$; intermediate corresponds to Sokal score of 0.8 to 1.2; and high corresponds to Sokal score of $>1.2$

Treatment-emergent AEs were reported by $96 \%$ of patients treated with bosutinib versus 95\% treated with imatinib (Table 2). Bosutinib, compared with imatinib, was associated with higher incidences of diarrhea ( $68 \% v 21 \%$, respectively), vomiting (32\% $v 13 \%$, respectively), and abdominal pain ( $11 \% \vee 5 \%$, respectively). Conversely, bosutinib, compared with imatinib, was associated with lower incidences of edema ( $11 \% v 38 \%$, respectively), bone pain ( $4 \% v 10 \%$, respectively), and muscle spasms ( $2 \% v 20 \%$, respectively). The aggregate incidence of grade 3 or 4 AEs was $64 \%$ in the bosutinib arm and $48 \%$ in the imatinib arm $(P<.001)$, with median durations of 13 and 15 days, respectively. Specific grade 3 or 4 nonhematologic AEs observed for more than $2 \%$ of patients included diarrhea (bosutinib, 11\%; imatinib, 1\%) and vomiting (bosutinib, 3\%; imatinib, 0\%; Table 2 ); all were grade 3 events in both treatment arms. Although diarrhea occurred in both treatment arms, most instances of diarrhea resolved (bosutinib, 85\%; imatinib, 77\%) by the time of this analysis. Per the protocol, it was recommended that at the first sign of diarrhea, medications such as diphenoxylate/atropine or loperamide should be used as needed, which effectively controlled diarrhea in most instances. Of those who experienced diarrhea with bosutinib, 67\% received concomitant medication for the management of diarrhea. The diarrhea events were frequently reported in the first 2 to 4 weeks from starting treatment but decreased over time among patients treated with bosutinib. Median time to onset was 3 days (range, 1 to 589 days) for bosutinib compared with 22.5 days (range, 1 to 476 days) for imatinib, and the median durations of a diarrhea event were 3 and 5 days, respectively. Of those who experienced diarrhea, dose interruptions and reductions for diarrhea were required by $21 \%$ and $8 \%$ of patients on bosutinib, respectively, versus $6 \%$ and $0 \%$ of patients on imatinib, respectively. Diarrhea was not the primary AE leading to treatment discontinuation for any patient on bosutinib, although one patient had concurrent diarrhea associated with GI toxicity (ie, vomiting) at the time of discontinuation.

Edema was less common (11\%) and occurred later (median, 113 days) with bosutinib compared with imatinib (38\%; median, 30 days). Seventy-eight percent of patients receiving bosutinib compared with $42 \%$ receiving imatinib had their edema resolve by the time of this analysis. No patient in either study arm discontinued treatment as a result of edema; three patients required dose interruption because of edema, with no recurrence on rechallenge.

Drug-related cardiac AEs were experienced by 10 patients (4\%) receiving bosutinib and eight patients (3\%) receiving imatinib. The most common drug-related cardiac AE was prolonged QT interval (bosutinib, $\mathrm{n}=5$ [one patient with grade 3]; imatinib, $\mathrm{n}=6$ ). The number of QT prolongations greater than 500 milliseconds with bosutinib versus imatinib was two (1\%) versus none for QTcF and two $(1 \%)$ versus one $(<1 \%)$ for QTcB, respectively.

Transient liver aminotransferase elevations (grade 3 or 4 ) were more common on treatment among patients treated with bosutinib versus imatinib (ALT, 22\% v 3\%, respectively; and AST, 11\% v 3\%, respectively; Table 2). The median time to onset for ALT and AST elevations was 28 days (for both) for bosutinib compared with 91 and 97 days, respectively, for imatinib. Dose interruptions and/or reductions were common among patients with aminotransferase elevations (bosutinib, 57\% and 36\%; imatinib, 24\% and 6\%, respectively), and many patients in both arms experienced multiple ALT/AST events (bosutinib, 71\%; imatinib, 53\%).

Grade 3 or 4 lipase elevation was observed in $7 \%$ of patients receiving bosutinib and 5\% receiving imatinib and was associated with pancreatitis in one patient on imatinib. The incidence of grade 3 or 4 laboratory abnormalities of anemia and thrombocytopenia was similar between treatment arms (anemia, 6\% for bosutinib and 7\% for imatinib; thrombocytopenia, 14\% each); however, grade 3 or 4 neutropenia was lower in the bosutinib arm (11\% v24\% for imatinib; Table 2).

\section{DISCUSSION}

Bosutinib did not demonstrate a superior rate of CCyR at 12 months versus imatinib based on the ITT population, which was the primary end point of the study. However, bosutinib treatment did demonstrate superiority in some secondary and exploratory efficacy end points. Bosutinib resulted in a superior rate of MMR at 12 months, 


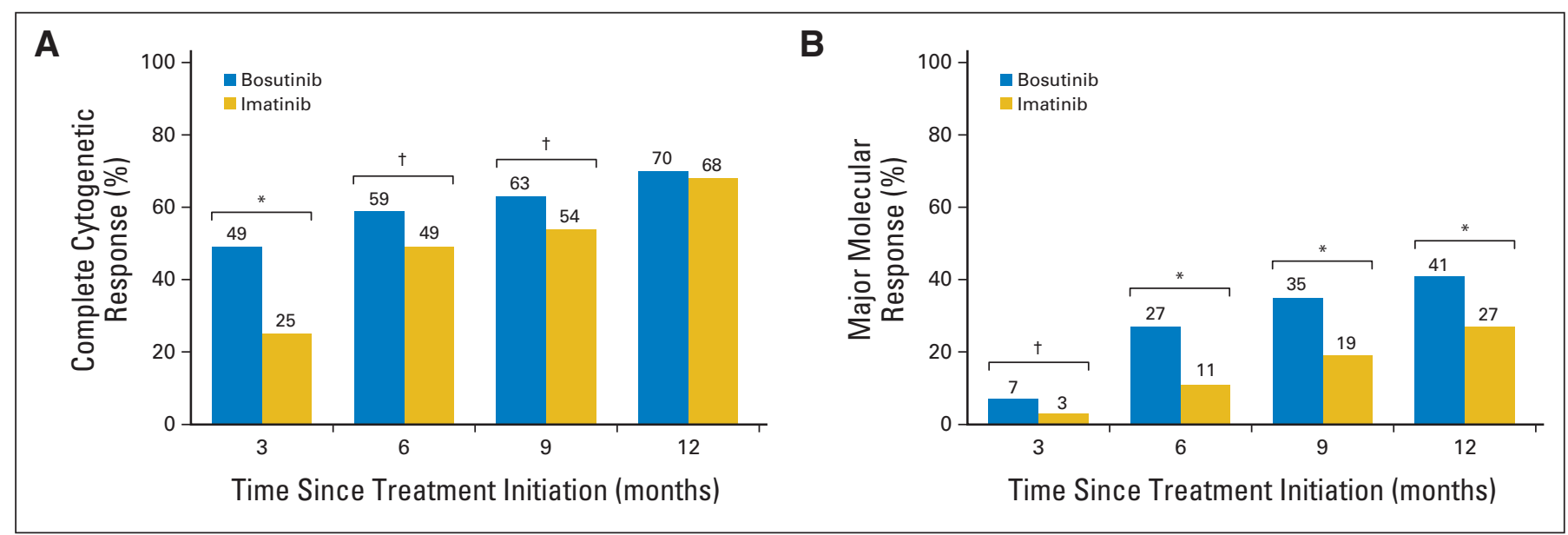

Fig 2. Rates of (A) complete cytogenetic response and (B) major molecular response in the intent-to-treat population. Cytogenetic analysis determined the presence of Philadelphia

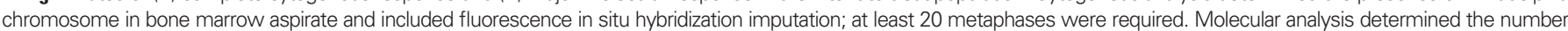

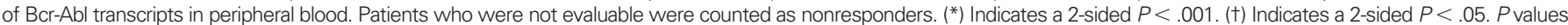

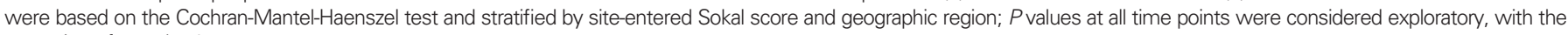
exception of month 12 .

which was the key secondary end point of the study. MMR is considered to represent approximately a 1-log deeper response than CCyR with respect to Bcr-Abl transcript levels and has been shown to predict longer duration of CCyR. ${ }^{21,22}$ The times to CCyR and MMR were shorter with bosutinib, which supports a more rapid debulking of tumor burden with bosutinib compared with standard-dose imatinib. Additionally, patients receiving bosutinib had a lower rate of disease progression/lack of efficacy (3\%) compared with patients receiving imatinib (10\%), including a trend toward fewer transformations to $\mathrm{AP} / \mathrm{BP} \mathrm{CML}(2 \% v 4 \%$, respectively). Together, these results indicate that bosutinib may be effective in some patients with newly diagnosed CP CML, even though the primary efficacy end point was not met. Continued follow-up will allow for long-term evaluation of bosutinib efficacy in this population.

There have also been two recent studies with the secondgeneration TKIs dasatinib and nilotinib versus imatinib in patients with CP CML in the first-line setting. ${ }^{5,6}$ Allowing for differences in study design, patient population, and the inherent difficulties of com-

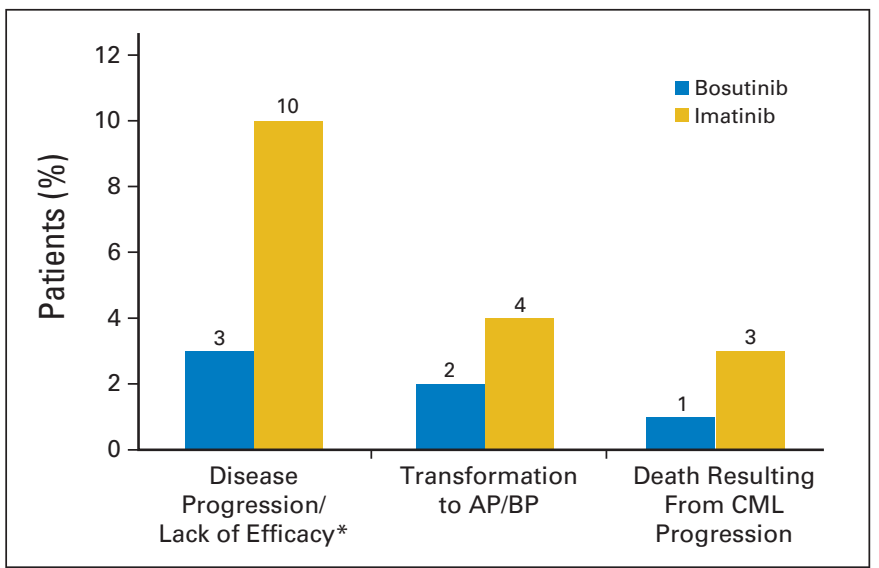

Fig 3. Rates of disease progression while on study treatment in the intent-totreat population. $\left({ }^{*}\right)$ Includes transformation to accelerated phase (AP)/blast phase (BP) chronic myeloid leukemia (CML). paring data across multiple studies, rates of CCyR by 12 months were similar among all three studies (bosutinib, 79\%; dasatinib, $83 \%$; nilotinib, $78 \%$ to $80 \%$ ), although the rate achieved with imatinib was slightly higher in the current study (75\%) than in the dasatinib and nilotinib studies ( $72 \%$ and $65 \%$, respectively), ${ }^{5,6}$ possibly because of differences in ITT population definitions and Sokal and Hasford risk groups across studies. The MMR rate at 12 months was higher in both the bosutinib versus imatinib and nilotinib versus imatinib studies (bosutinib, $41 \%$; nilotinib, $43 \%$ to $44 \%$; imatinib, $27 \%$ and $22 \%$, respectively), ${ }^{6}$ and the rate of MMR by 12 months was also higher with bosutinib and dasatinib across studies (bosutinib, 47\%; dasatinib, $46 \%$; imatinib, $32 \%$ and $28 \%$, respectively). ${ }^{5}$ In addition, all three second-generation TKIs were associated with faster achievement of CCyR and MMR compared with imatinib. ${ }^{5,6}$ Similar rates of transformation to AP/BP CML were observed in all three studies (bosutinib, $2 \%$; dasatinib, $2 \%$; nilotinib, $1 \%$; imatinib, $4 \%, 3 \%$, and $4 \%) ., 5$

Overall, bosutinib was associated with a toxicity profile distinct from that of imatinib. However, an unexpectedly high number of patients ( $\mathrm{n}=$ $48,19 \%$ ) were discontinued from bosutinib treatment as a result of an $\mathrm{AE}$, including 15 (31\%) of 48 patients who discontinued before their first postbaseline assessment. These data require confirmation in future studies. Thus, early discontinuations as a result of toxicity may have contributed to the lower rate of CCyR associated with bosutinib in the ITT population, which included these patients as nonresponders.

Although bosutinib therapy was associated with greater rates of diarrhea, vomiting, and aminotransferase elevations compared with imatinib, most instances were transient and, in the case of diarrhea, frequently self-limiting after the first 2 to 3 months of therapy. Fluid retention, which has commonly been observed with dasatinib $b^{5,17,23-28}$ and imatinib, ${ }^{5,6,27,29}$ occurred infrequently with bosutinib. Despite the occurrence of liver function test abnormalities with bosutinib treatment, no cases were associated with permanent liver injury. Low rates of grade 3 or 4 (all causality) anemia (6\%), neutropenia (11\%), and thrombocytopenia (14\%) were reported with bosutinib. The minimal inhibition of c-KIT by bosutinib may have contributed to the low incidence of myelosuppressive events, particularly grade 3 or 4 


\begin{tabular}{|c|c|c|c|c|c|c|c|c|}
\hline \multirow[b]{3}{*}{ Event } & \multicolumn{4}{|c|}{ Bosutinib $(n=248)$} & \multicolumn{4}{|c|}{ Imatinib $(n=251)$} \\
\hline & \multicolumn{2}{|c|}{ All Grades } & \multicolumn{2}{|c|}{ Grade 3 or 4} & \multicolumn{2}{|c|}{ All Grades } & \multicolumn{2}{|c|}{ Grade 3 or 4} \\
\hline & No. of Patients & $\%$ & No. of Patients & $\%$ & No. of Patients & $\%$ & No. of Patients & $\%$ \\
\hline \multicolumn{9}{|c|}{$\begin{array}{l}\text { Adverse events reported for } \geq 10 \% \text { of } \\
\text { patients with any grade }\end{array}$} \\
\hline Diarrhea & 168 & 68 & 26 & 11 & 52 & 21 & 2 & 1 \\
\hline Vomiting & 78 & 32 & 8 & 3 & 33 & 13 & 0 & 0 \\
\hline Nausea & 76 & 31 & 2 & 1 & 87 & 35 & 0 & 0 \\
\hline Rash & 50 & 20 & 3 & 1 & 38 & 15 & 2 & 1 \\
\hline Pyrexia & 40 & 16 & 2 & 1 & 23 & 9 & 3 & 1 \\
\hline Upper abdominal pain & 30 & 12 & 0 & 0 & 13 & 5 & 0 & 0 \\
\hline Abdominal pain & 29 & 11 & 3 & 1 & 12 & 5 & 0 & 0 \\
\hline Edema* & 27 & 11 & 1 & $<1$ & 95 & 38 & 2 & 1 \\
\hline Fatigue & 27 & 11 & 3 & 1 & 29 & 12 & 2 & 1 \\
\hline Headache & 25 & 10 & 2 & 1 & 21 & 8 & 0 & 0 \\
\hline Upper respiratory infection & 25 & 10 & 0 & 0 & 16 & 6 & 0 & 0 \\
\hline Bone pain & 9 & 4 & 0 & 0 & 26 & 10 & 2 & 1 \\
\hline Muscle cramps & 6 & 2 & 0 & 0 & 50 & 20 & 0 & 0 \\
\hline \multicolumn{9}{|c|}{$\begin{array}{l}\text { Laboratory abnormalities reported for } \geq 5 \% \\
\text { of patients with grade } 3 \text { or } 4\end{array}$} \\
\hline Anemia & 200 & 81 & 16 & 6 & 211 & 84 & 17 & 7 \\
\hline Thrombocytopenia & 163 & 66 & 35 & 14 & 157 & 63 & 36 & 14 \\
\hline Hypophosphatemia & 116 & 47 & 10 & 4 & 159 & 63 & 38 & 15 \\
\hline Elevated ALT & 170 & 69 & 55 & 22 & 74 & 29 & 8 & 3 \\
\hline Elevated AST & 140 & 56 & 26 & 11 & 68 & 27 & 8 & 3 \\
\hline Neutropenia & 70 & 28 & 27 & 11 & 135 & 54 & 60 & 24 \\
\hline Elevated lipase & 94 & 38 & 22 & 9 & 85 & 34 & 12 & 5 \\
\hline
\end{tabular}

neutropenia. ${ }^{14}$ Overall, there were fewer deaths in the bosutinib arm, with no treatment-related deaths and few CML-related deaths in both treatment arms. The trends in toxicity profile observed for bosutinib in the current study are similar to those previously observed for bosutinib in CP CML after failure of imatinib treatment. ${ }^{15}$

In conclusion, bosutinib did not achieve the primary efficacy end point of a superior rate of CCyR at 12 months versus imatinib; however, bosutinib did demonstrate other potential benefits in patients with newly diagnosed CP CML. In addition, the safety profile of bosutinib was distinct from that of imatinib. Further follow-up is needed to provide long-term data on duration of response, transformation to AP/BP CML, and overall survival, as well as the tolerability profile of bosutinib in newly diagnosed CP CML.

\section{AUTHORS' DISCLOSURES OF POTENTIAL CONFLICTS}

$$
\text { OF INTEREST }
$$

Although all authors completed the disclosure declaration, the following author(s) and/or an author's immediate family member(s) indicated a financial or other interest that is relevant to the subject matter under consideration in this article. Certain relationships marked with a " $U$ " are those for which no compensation was received; those relationships marked with a " $C$ " were compensated. For a detailed description of the disclosure categories, or for more information about ASCO's conflict of interest policy, please refer to the Author Disclosure Declaration and the Disclosures of Potential Conflicts of Interest section in Information for Contributors.

Employment or Leadership Position: Christine Powell, Pfizer (C); Karïn Gogat, Pfizer (C); Athena M. Countouriotis, Pfizer (C) Consultant or
Advisory Role: Jorge E. Cortes, Pfizer (C), Bristol-Myers Squibb (C), Novartis (C); Hagop M. Kantarjian, Novartis (C); Tim H. Brümmendorf, Pfizer (C), Bristol-Myers Squibb (C), Novartis (C); Laimonas Griskevicius, Novartis (C); Carlo Gambacorti-Passerini, Pfizer (C), Bristol-Myers Squibb (C) Stock Ownership: Christine Powell, Pfizer; Athena M. Countouriotis, Pfizer Honoraria: Dong-Wook Kim, Bristol-Myers Squibb, Novartis; Tim H. Brümmendorf, Pfizer, Bristol-Myers Squibb, Novartis; Laimonas Griskevicius, Novartis; Carlo Gambacorti-Passerini, Pfizer, Bristol-Myers Squibb Research Funding: Jorge E. Cortes, Pfizer, Bristol-Myers Squibb, Novartis; Hagop M. Kantarjian, Pfizer, Bristol-Myers Squibb, Novartis; Tim H. Brümmendorf, Novartis; Laimonas Griskevicius, Novartis; Carlo Gambacorti-Passerini, Wyeth Research/Pfizer Expert Testimony: None Other Remuneration: Laimonas Griskevicius, Novartis

\section{AUTHOR CONTRIBUTIONS}

Conception and design: Jorge E. Cortes, Tim H. Brümmendorf, Athena M. Countouriotis, Carlo Gambacorti-Passerini

Provision of study materials or patients: Jorge E. Cortes, Dong-Wook Kim, Hagop M. Kantarjian, Tim H. Brümmendorf, Irina Dyagil, Laimonas Griskevicius, Hemant Malhotra, Carlo Gambacorti-Passerini Collection and assembly of data: Jorge E. Cortes, Dong-Wook Kim, Tim H. Brümmendorf, Irina Dyagil, Laimonas Griskevicius, Hemant Malhotra, Christine Powell, Karïn Gogat, Athena M. Countouriotis

Data analysis and interpretation: Jorge E. Cortes, Dong-Wook Kim, Hagop M. Kantarjian, Tim H. Brümmendorf, Christine Powell, Karïn Gogat, Athena M. Countouriotis, Carlo Gambacorti-Passerini Manuscript writing: All authors Final approval of manuscript: All authors 


\section{REFERENCES}

1. National Comprehensive Cancer Network: NCCN clinical practice guidelines in oncology: Chronic myelogenous leukemia-Version 2.2011. http://mww.nccn.org/ professionals/physician_gls/PDF/cml.pdf

2. Druker BJ, Guilhot F, O'Brien SG, et al: Fiveyear follow-up of patients receiving imatinib for chronic myeloid leukemia. N Engl J Med 355:24082417, 2006

3. O'Brien $S G$, Guilhot F, Larson RA, et al: Imatinib compared with interferon and low-dose cytarabine for newly diagnosed chronic-phase chronic myeloid leukemia. N Engl J Med 348:994-1004, 2003

4. Hehlmann R, Lauseker M, Jung-Munkwitz S, et al: Tolerability-adapted imatinib $800 \mathrm{mg} / \mathrm{d}$ versus $400 \mathrm{mg} / \mathrm{d}$ versus $400 \mathrm{mg} / \mathrm{d}$ plus interferon-alpha in newly diagnosed chronic myeloid leukemia. J Clin Oncol 29:1634-1642, 2011

5. Kantarjian $H$, Shah NP, Hochhaus $A$, et al: Dasatinib versus imatinib in newly diagnosed chronic-phase chronic myeloid leukemia. N Engl $J$ Med 362:2260-2270, 2010

6. Saglio G, Kim DW, Issaragrisil S, et al: Nilotinib versus imatinib for newly diagnosed chronic myeloid leukemia. N Engl J Med 362:2251-2259, 2010

7. Cortes JE, Jones D, O'Brien S, et al: Nilotinib as front-line treatment for patients with chronic myeloid leukemia in early chronic phase. $\mathrm{J}$ Clin Oncol 28:392-397, 2010

8. Cortes JE, Jones $D, O$ 'Brien $S$, et al: Results of dasatinib therapy in patients with early chronicphase chronic myeloid leukemia. J Clin Oncol 28: 398-404, 2010

9. Rosti G, Palandri F, Castagnetti F, et al: Nilotinib for the frontline treatment of $\mathrm{Ph}(+)$ chronic myeloid leukemia. Blood 114:4933-4938, 2009

10. Puttini M, Coluccia AM, Boschelli F, et al: In vitro and in vivo activity of SKI-606, a novel Src-Abl inhibitor, against imatinib-resistant $\mathrm{Bcr}-\mathrm{Abl}+$ neoplastic cells. Cancer Res 66:11314-11322, 2006
11. Golas JM, Arndt K, Etienne C, et al: SKI-606, a 4-anilino-3-quinolinecarbonitrile dual inhibitor of Src and $\mathrm{Abl}$ kinases, is a potent antiproliferative agent against chronic myelogenous leukemia cells in culture and causes regression of K562 xenografts in nude mice. Cancer Res 63:375-381, 2003

12. Remsing Rix LL, Rix U, Colinge J, et al: Global target profile of the kinase inhibitor bosutinib in primary chronic myeloid leukemia cells. Leukemia 23:477-485, 2009

13. Konig $\mathrm{H}$, Holyoake $T L$, Bhatia R: Effective and selective inhibition of chronic myeloid leukemia primitive hematopoietic progenitors by the dual Src/ Abl kinase inhibitor SKI-606. Blood 111:2329-2338, 2008

14. Bartolovic $K$, Balabanov S, Hartmann U, et al: Inhibitory effect of imatinib on normal progenitor cells in vitro. Blood 103:523-529, 2004

15. Cortes JE, Kantarjian HM, Brümmendorf $\mathrm{TH}$ et al: Safety and efficacy of bosutinib (SKI-606) in chronic phase Philadelphia chromosome-positive chronic myeloid leukemia patients with resistance or intolerance to imatinib. Blood 118:4567-4576, 2011

16. Khoury HJ, Cortes JE, Kantarjian HM, et al: Bosutinib is active in chronic phase chronic myeloid leukemia after imatinib and dasatinib and/or nilotinib therapy failure. Blood 119:3403-3412, 2012

17. Talpaz M, Shah NP, Kantarjian H, et al: Dasatinib in imatinib-resistant Philadelphia chromosomepositive leukemias. N Engl J Med 354:2531-2541, 2006

18. Kantarjian HM, Giles F, Gattermann N, et al: Nilotinib (formerly AMN107), a highly selective BCR$A B L$ tyrosine kinase inhibitor, is effective in patients with Philadelphia chromosome-positive chronic myelogenous leukemia in chronic phase following imatinib resistance and intolerance. Blood 110:3540-3546, 2007

19. Hochhaus A, Baccarani M, Deininger $M$, et al: Dasatinib induces durable cytogenetic responses in patients with chronic myelogenous leukemia in chronic phase with resistance or intolerance to imatinib. Leukemia 22:1200-1206, 2008
20. Baccarani M, Cortes J, Pane F, et al: Chronic myeloid leukemia: An update of concepts and management recommendations of European LeukemiaNet. J Clin Oncol 27:6041-6051, 2009

21. Cortes J, Talpaz M, O'Brien S, et al: Molecular responses in patients with chronic myelogenous leukemia in chronic phase treated with imatinib mesylate. Clin Cancer Res 11:3425-3432, 2005

22. Iacobucci I, Saglio G, Rosti G, et al: Achieving a major molecular response at the time of a complete cytogenetic response (CCgR) predicts a better duration of $\mathrm{CCgR}$ in imatinib-treated chronic myeloid leukemia patients. Clin Cancer Res 12:3037-3042, 2006

23. Hochhaus A, Kantarjian HM, Baccarani M, et al: Dasatinib induces notable hematologic and cytogenetic responses in chronic-phase chronic myeloid leukemia after failure of imatinib therapy. Blood 109:2303-2309, 2007

24. Kantarjian $H$, Pasquini $R$, Hamerschlak $N$, et al: Dasatinib or high-dose imatinib for chronic-phase chronic myeloid leukemia after failure of first-line imatinib: A randomized phase 2 trial. Blood 109: 5143-5150, 2007

25. Shah NP, Kantarjian HM, Kim DW, et al: Intermittent target inhibition with dasatinib $100 \mathrm{mg}$ once daily preserves efficacy and improves tolerability in imatinib-resistant and -intolerant chronicphase chronic myeloid leukemia. J Clin Oncol 26: 3204-3212, 2008

26. Quintás-Cardama $A$, Kantarjian $H$, O’Brien $S$ et al: Pleural effusion in patients with chronic myelogenous leukemia treated with dasatinib after imatinib failure. J Clin Oncol 25:3908-3914, 2007

27. Gora-Tybor J, Robak T: Targeted drugs in chronic myeloid leukemia. Curr Med Chem 15:30363051, 2008

28. Bristol-Myers Squibb: Sprycel (Dasatinib) tablet for oral use [package insert]. Princeton, NJ, Bristol-Myers Squibb, 2009

29. Novartis: Gleevec (Imatinib Mesylate) tablets for oral use [package insert]. East Hanover, NJ Novartis, 2009 